\title{
SURGICAL TREATMENT OF MOYAMOYA DISEASE IN CHILDREN
}

\author{
Zeferino Demartini Jr', Rodrigo Tomazini Martins ${ }^{2}$, \\ Carlos Eduardo Dall'Aglio Rocha', Luiz Afonso Dias Matos', \\ Antonio Ronaldo Spotti $i^{5}$, Waldir Antônio Tognola ${ }^{6}$, Márcio Luiz Tostes dos Santos ${ }^{7}$
}

The chronic occlusive cerebrovascular disease was first described in 1957 by Takeuchi and Shimizu as hypoplasia of bilateral internal carotid arteries (ICA), ${ }^{1,2}$. Later it was considered acquired and progressive, due to stenosis or occlusion of the arteries next to the circle of Willis, with abnormal arteries as collaterals ${ }^{1-5}$. In 1967, Suzuki and Takaku introduced the term moyamoya, Japanese word that means hazy, due to the image similar to a puff of smoke at angiography ${ }^{1,2,4,5}$. It is a rare disorder that presents mostly ischemic symptoms in children and hemorrhage in adults ${ }^{1-3,5}$. The disease induces the formation of a new vascular network (rete mirabilis)' ${ }^{2}$ We report two cases of the moyamoya disease in children surgically treated.

\section{CASE}

\section{Case 1}

EKT, 5 years old, male, Japanese origin, born in São José do Rio Preto-SP, Brazil, living in Japan. By the age of 4 presented frequent episodes of transient right side hemiparesis, recovering spontaneously. Cerebral angiography performed in Japan diagnosed moyamoya disease and then he returned to Brazil. He arrived to the Hospital de Base with normal neurological exam. Other cerebral angiography was done (Fig 1), indicating stenosis of the right ICA, left middle cerebral artery (MCA), bilateral anterior cerebral arteries (ACA) and abnormal vessels by ophthalmic artery, as well as preeminent posterior choroid arteries with anastomosis by the ventricles. He was undergone surgical treatment with encephaloduroarteriosynangiosis (EDAS). The left temporal superficial artery (TSA) was sewn to the pia mater at the lateral fissure close to the MCA using 8.0 mononylon. A flap of dura mater and a branch of middle meningeal artery (MMA) were placed over the cortex, and duroplasty was made with galeal flap. Angiographic study six months after showed fair

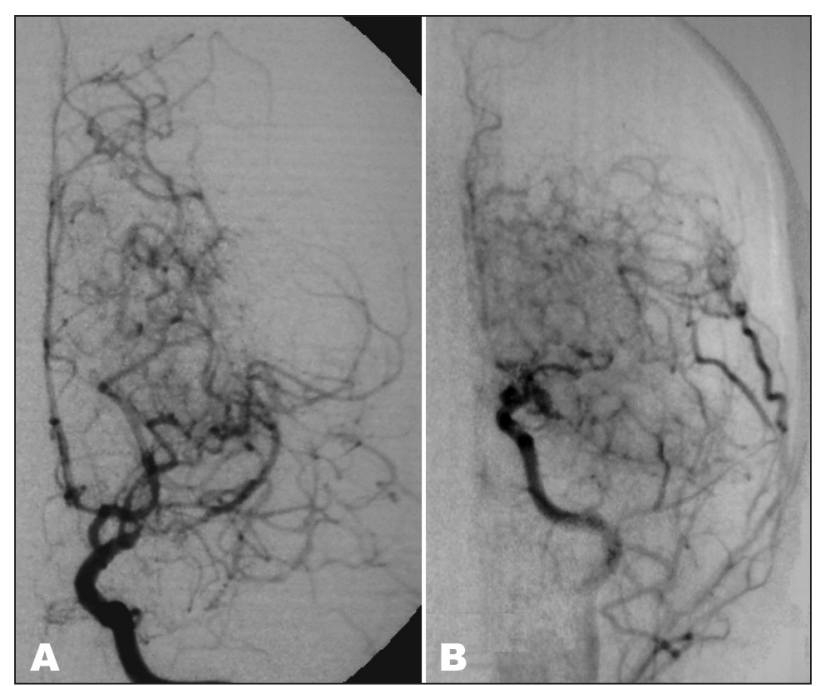

Fig 1. Antero-posterior images of cerebral angiography of the case 1 shows stenosis in left ICA, ACA and MCA (A); post-operative image of the CCA shows filling of the MCA through ECA (B). (ICA: internal carotid artery; $A C A$ : anterior cerebral artery; MCA middle cerebral artery; CCA: common carotid artery; ECA: external carotid artery; MIP: maximum intensity projection).

filling of the left MCA, classified as B in the Matsushima grading scale ${ }^{6}$. There were three mild transient ischemic attacks (TIA) in a period of six months, characterizing good outcome.

\section{Case 2}

LSN, 7 years old, female, born in Sud Menucci-SP, Brazil. At 6 years old, she presented disarthrya and right side paresis and paresthesia, with spontaneous recovery after two days. A new ischemic episode occurred four months later maintaining disarthrya and right paresis. Acetyl salicylic acid (ASA) 100mg per day was started, and brought to the Hospital de Base. Brain MR

\section{TRATAMENTO CIRÚRGICO DA DOENÇA DE MOYAMOYA EM CRIANÇAS}

Unidade de Neurocirurgia Endovascular do Serviço de Neurocirurgia do Hospital de Base de São José do Rio Preto, São José do Rio Preto, SP, Brazil: 'MD, Neurosurgeon, fellow of Unit of Endovascular Neurosurgery at Hospital de Base de São José do Rio Preto, SP, Brazil; ${ }^{2}$ MD, Medical student at Faculdade de Medicina de Marília, SP, Brazil; ${ }^{3} \mathrm{MD}$, Neurosurgeon at Hospital de Base de São José do Rio Preto, SP, Brazil; ${ }^{4} \mathrm{MD}$, Neurosurgeon, resident of Unit of Endovascular Neurosurgery at Hospital de Base de São José do Rio Preto, SP, Brazil; ${ }^{5} \mathrm{MD}$, PhD, Head of the Department of Neurological Sciences at Faculdade de Medicina de São José do Rio Preto, SP, Brazil; ${ }^{6}$ MD, PhD, Professor of the Department of Neurological Sciences at Faculdade de Medicina de São José do Rio Preto, SP, Brazil; ${ }^{7}$ MD, Head of the Unit of Endovascular Neurosurgery at Hospital de Base de São José do Rio Preto, SP, Brazil.

Received 25 September 2007, received in final form 14 January 2008. Accepted 25 February 2008.

Dr. Zeferino Demartini Jr - Hospital de Base / Unidade de Neurocirurgia Endovascular - Avenida Brig. Faria Lima 5544 - $15090-000$ São José do Rio Preto SP - Brasil. E-mail: demartini9@yahoo.com 

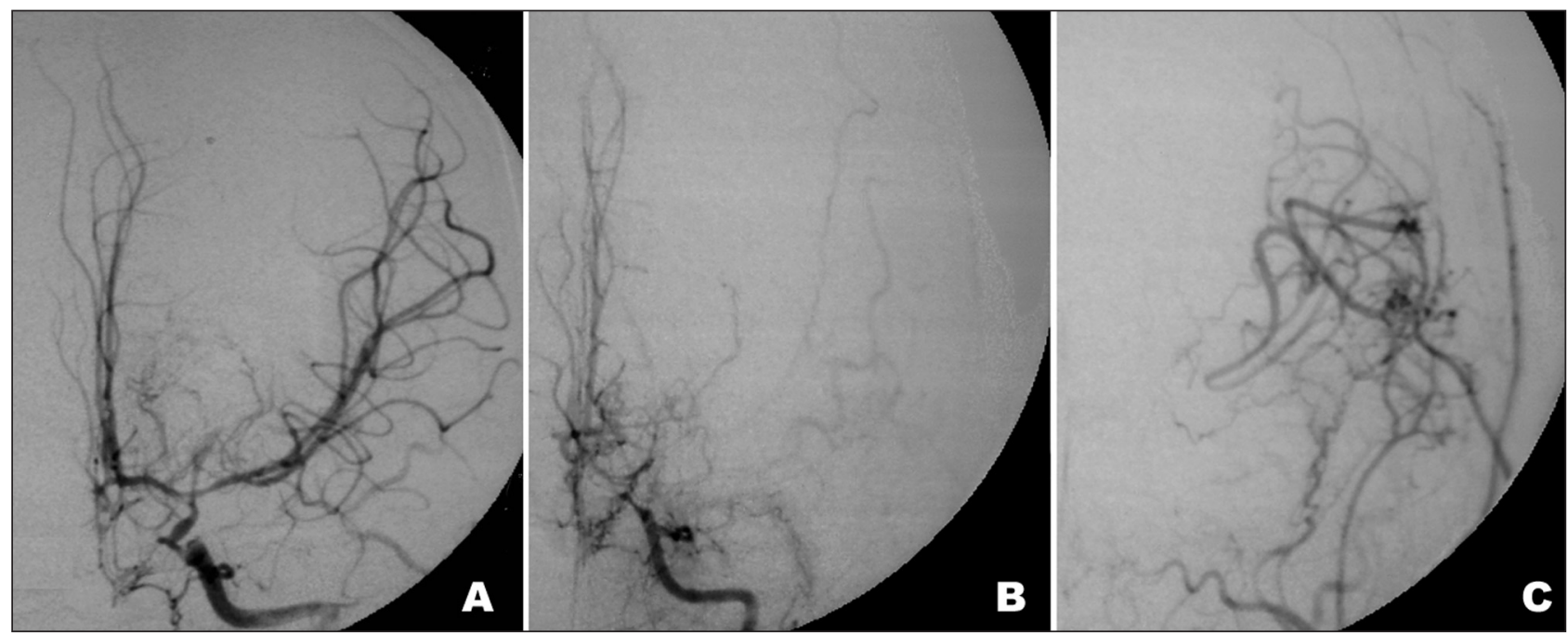

Fig 2. Images of cerebral angiography and MR of the case 2 shows stenosis of the left ICA, MCA and ACA (A); post-operative angiography shows reduced flow in MCA through ICA (B), with filling of the MCA branches through ECA (C). (ICA: internal carotid artery; ACA: anterior cerebral artery; MCA middle cerebral artery; CCA: common carotid artery; ECA: external carotid artery; MIP: maximum intensity projection)

showed multiple chronic ischemic lesions and MRA revealed multiple intracranial stenosis. Anti-fosfolipids antibodies and haemoglobin electrophoresis were normal. Angiographic study demonstrated bilateral stenosis on the supraclinoid branches of the ICA, extended to ACA and MCA, with anastomosis by the posterior cerebral arteries and ECA, with typical moyamoya vessels (Fig 2). The EDAS was performed by the same technique of the case 1. Post operative was uneventful, and the symptoms disappeared for seven months of follow-up, with approve of the speech but maintained hemiparesis. MR performed after 6 months showed cerebral atrophy in left hemisphere, worsen stenosis at the ICA and anastomosis patency. The outcome was considered good.

\section{DISCUSSION}

The moyamoya disease is more prevalent in the Asian population ${ }^{1-3}$, with a 0.35 per 100.000 inhabitants incidence in the Japan 1,2,5. Although recent studies demonstrate lower rates in the Caucasians ${ }^{1,2}$. In female gender it occurs mostly ${ }^{2}$ at a rate of 1.8:1,4,5. There is a bimodal peek, in the first and around the fourth decades of life $\mathrm{e}^{1,2,5}$.

Despite its unknown etiology $y^{1,2,4,5}$, it is characterized by bilateral thickening of the intima layer at terminal portions of the $\mathrm{ICA}^{1,4,5}$. In these regions the occlusion is slow and progressive, developing a new vascular network from the perforators, choroid and branches of the external carotid arteries $(\mathrm{ECA})^{2,5}$.

According to the Research Committee on Spontaneous Occlusion of the Circle of Willis (moyamoya disease), for the diagnose the patient must present bilateral occlusion and/or stenosis of the ICA terminal branches and/or proximal ACA and MCA branches, besides the formation of new vascular network around the circle of Willis, and no other diseases with similar characteristics ${ }^{1,4}$.

Cerebral angiography is considered gold standard for diagnosis, evaluate and study the vascular anatomy and plan the surgical treatment ${ }^{7}$. New vascular formations corresponding to the typical pattern of puff of smoke is also showed $^{1,2,5}$.

The natural history of moyamoya disease is poor, coursing with progressive neurological deterioration ${ }^{8}$. As its etiology is unknown ${ }^{1,2,5}$, there is no definite treatment so $\mathrm{far}^{5}$. Most of them in acute phase are symptomatic, with the aim of not developing further sequels and stop the progression of the lesions ${ }^{1,5}$. Observant, clinical or surgical treatment may be indicated. Pharmacological treatment of ischemia is based on palliative cares such as anticoagulation and antiplatelets ${ }^{2,4,5}$, but may be unefficient ${ }^{2-4}$.

Surgery offers the best results and benefits for the patients $^{1,2,4}$. Repeated TIA or strokes, worst symptoms, progressive mental retardation, low blood flow to the brain documented by angiography, cerebral atrophy on CT are indication to revascularization procedure ${ }^{3,5}$. Several techniques are proposed ${ }^{3,6,9}$. Direct bypass refers to TSA-MCA anastomosis ${ }^{6,9}$, while indirect consists on placing a flap of dura mater (encephaloduroarteriosynangiosis, EDAS), galea (encephalogaleosynangiosis, EGS) or muscle (encephalomyosynangiosis, EMS) ${ }^{9}$ over the cerebral cortex. Those techniques may be combined in a same patient $\mathrm{t}^{1,3,4,6}$ and the goal remains on minimize or halt the cerebral ischemic progression, reverting or reducing the clinical symptoms ${ }^{1,3,4,6}$. Direct bypass provides higher blood flow immediately but present more technical difficulties to the surgeon and risk to the patient, due to the blood 
stasis and the small size of the arteries involved ${ }^{3,9}$. Indirect bypass has no statistical differences in symptomatic outcome ${ }^{3}$. This procedure is easier and safer to perform, becoming more popular especially in children ${ }^{3,9}$. The technique chosen (EDAS), with TSA and MMA placed over the cortex aimed maximize the reperfusion of the ischemic areas induced by angiogenesis ${ }^{4,9}$. Pial synangiosis allows the development of new collaterals, because arachnoid membrane can act as a barrier ${ }^{9,10}$. Prognosis after surgery in children is good, and TIA tend to disappear in few months ${ }^{1,3,4}$. Both patients had collateral formation at cerebral angiography classified in fair and good outcomes in the Matsushita grading scale $e^{6}$, suggesting compensation of the ischemia. Long-term follow up is necessary to access the effectiveness of the procedures.

\section{REFERENCES}

1. Fukui M, Kono S, Sueishi K, Ikezaki K. Moyamoya disease. Neuropathology 2000;20(Suppl):S61-S64.
2. Franco C, Fukujima M, de Oliveira R, Gabbai A. Moyamoya disease: report of three cases in Brazilian patients. Arq Neuropsiquiatr 1999;57:371-376

3. Fung L, Thompson D, Ganesan V. Revascularisation surgery for paediatric moyamoya: a review of the literature. Childs Nerv Syst 2005;21:358-364.

4. Tripathi P, Tripathi V, Naik R, Patel J. Moya Moya cases treated with encephaloduroarteriosynangiosis. Indian Pediatr 2007;44:123-127.

5. Fukui M. Guidelines for the diagnosis and treatment of spontaneous occlusion of the circle of Willis ('Moyamoya' disease). Members of the Research Committee on Spontaneous Occlusion of the Circle of Willis (Moyamoya Disease) of the Ministry of Health and Welfare, Japan. Clin Neurol Neurosurg 1997;99(Suppl 2):S238-S240.

6. Matsushima T, Inoue T, Suzuki S, Fujii K, Fukui M, Hasuo K. Surgical treatment of moyamoya disease in pediatric patients: comparison between the results of indirect and direct revascularization procedures. Neurosurgery 1992;31:401-405

7. Punt J. Surgical management of paediatric stroke. Pediatr Radiol 2004; 34:16-23.

8. Ueki K, Meyer F, Mellinger J. Moyamoya disease: the disorder and surgical treatment. Mayo Clin Proc 1994;69:749-757.

9. Kinugasa K, Mandai S, Kamata I, Sugiu K, Ohmoto T. Surgical treatment of moyamoya disease: operative technique for encephalo-duroarterio-myo-synangiosis, its follow-up, clinical results, and angiograms. Neurosurgery 1993;32:527-531.

10. Adelson P, Scott R. Pial synagiosis for moyamoya syndrome in children. Pediatr Neurosurg 1995;23:26-33. 